\title{
IDENTIFICANDO AS MELHORES PRÁTICAS OPERACIONAIS ATRAVÉS DA ASSOCIAÇÃO BENCHMARKING-DEA: O CASO DAS REFINARIAS DE PETRÓLEO
}

\author{
Vinícius Albuquerque Vasconcellos * \\ Coord. de Progr. de Pós-Grad. em Engenharia (COPPE) \\ Universidade Federal do Rio de Janeiro (UFRJ) \\ Rio de Janeiro - RJ \\ nicius@openlink.com.br
}

\author{
Alberto Gabbay Canen \\ Programa de Engenharia de Produção / COPPE \\ Universidade Federal do Rio de Janeiro (UFRJ) \\ Rio de Janeiro - RJ \\ agcanen@pep.ufrj.br \\ Marcos Pereira Estellita Lins \\ Programa de Engenharia de Produção / COPPE \\ Universidade Federal do Rio de Janeiro (UFRJ) \\ Rio de Janeiro - RJ \\ lins@pep.ufrj.br
}

* Corresponding author/autor para quem as correspondências devem ser encaminhadas

Recebido em 05/2004; aceito em 10/2005 após 3 revisões

Received May 2004; accepted October 2005 after 3 revisions

\begin{abstract}
Resumo
Este trabalho estuda a implementação de um método de Pesquisa Operacional denominado Análise Envoltória de Dados (DEA) em uma etapa de um processo de benchmarking, para identificar e analisar as melhores práticas operacionais de um modelo representativo de um sistema de unidades produtivas e, em função dessas análises, sugerir um plano de medidas para o alcance da eficiência máxima. A atuação do conjunto Benchmarking-DEA é observada em um estudo de caso aplicado em um grupo de refinarias de uma empresa de petróleo multinacional.
\end{abstract}

Palavras-chave: benchmarking; DEA; refinarias de petróleo; medição de desempenho.

\begin{abstract}
This work studies the implementation of an Operational Research method named Data Envelopment Analysis (DEA) in a stage of a benchmarking process, in order to identify and evaluate the operational best practices of a representative model of productive units system and, through these analysis, to suggest an action plan to reach the maximum efficiency. The Benchmarking-DEA association behaviour is observed in a case study applied in a set of refineries of a multinational petroleum company.
\end{abstract}

Keywords: benchmarking; DEA; petroleum refineries; performance measurement. 


\section{Introdução}

Medidas de eficiência têm tradicionalmente sido uma preocupação gerencial tanto nas indústrias de bens quanto nas empresas prestadoras de serviços. A literatura apresenta uma ampla variedade de métodos usados para medir eficiência. Aproximações por curvas de fronteiras medem produtividade contra funções de produção. Uma função de produção define os máximos níveis de outputs atingíveis com uma certa combinação de inputs ou o mínimo nível possível de inputs para ser usado na produção de um certo nível de outputs. A produtividade é definida pela engenharia como uma comparação entre a performance atual de um sistema e um conjunto adequado de padrões pré-definidos.

A preocupação deste estudo vai além disso, já que o foco principal não é exatamente mensurar a produtividade de uma empresa de um certo setor, mas sim detectar aquela que opera com maior eficiência seus inputs e outputs definidos por um modelo, cuja principal característica é a multidisciplinaridade de seus parâmetros, fator imprescindível no escopo de uma prática de benchmarking. Segundo Homburg (2001), a principal idéia do método DEA (Data Envelopment Analysis - Análise Envoltória de Dados) é estender o tradicional conceito de produtividade ou eficiência para torná-lo adequado ao formato multi-input $\mathrm{e}$ multi-output. Essa idéia resume o emprego de um estudo de DEA no presente trabalho, pois este método se aproxima da filosofia benchmarking.

O emprego de táticas de gerenciamento de falhas e a utilização de ferramentas de análise inapropriadas podem ser evitados através do uso métodos de benchmarking integrados em que diversas unidades de trabalho são examinadas simultaneamente para identificar as melhores práticas. Soluções mais reais são produzidas e/ou sinergias potenciais são reveladas. Em particular, a metodologia de Análise Envoltória de Dados tem provado ser uma poderosa ferramenta para o benchmarking na identificação das fronteiras eficientes (Ross \& Droge, 2002). Post \& Spronk (1999) afirmam que, como a aplicação do método DEA é focada em observações de práticas operacionais de uma amostragem de unidades comparáveis, as unidades apontadas como referência apresentam um maior apelo prático e funcionam de maneira mais justa do que padrões normativos de engenharia. Neste sentido, DEA é um método matemático cuja concepção vai de encontro ao que se deseja alcançar no presente estudo, que é um indicador de desempenho quantitativo para a detecção das melhores práticas de um determinado segmento de atividade.

O presente estudo de caso visa verificar o comportamento de um modelo criado para funcionar como um indicador de desempenho operacional para benchmarking, através da aplicação do método DEA, para as refinarias de petróleo de uma empresa multinacional deste segmento, com base em suas eficiências de operação. Busca-se detectar com isso, a unidade (ou grupo de unidades) que opera mais eficientemente seus recursos. $\mathrm{O}$ indicador de desempenho proposto no estudo, portanto, tem a função de estabelecer a unidade referência neste tipo de mercado, ou seja, aquela que apresenta as melhores práticas operacionais, assim como o diagnóstico de desempenho das unidades ineficientes.

\section{Benchmarking}

Segundo Spendolini (1994), benchmarking é um processo contínuo e sistemático para avaliar produtos, serviços e processos de trabalho de organizações que são reconhecidas como representantes das melhores práticas, com a finalidade de melhoria organizacional. O processo de benchmarking abordado neste estudo tem caráter quantitativo. Trata-se de um 
conjunto de práticas que visa atingir metas quantitativas ou mensuráveis a partir de dados coletados das empresas que apresentam as melhores práticas de uma determinada atividade. A capacidade de quantificar pode ser usada para apoiar as atividades e as decisões gerenciais.

O diagrama de fluxo do processo de benchmarking é detalhado, de acordo com a seguinte seqüência:

i. Busca das referências (levantamento dos detentores das melhores práticas);

ii. Procedimento de coleta de dados;

iii. Análise dos resultados. Proposta de utilização da técnica DEA;

iv. Implementação dos resultados.

Um dos fatores determinantes para o emprego da metodologia DEA como um indicador de performance para o benchmarking quantitativo, é exatamente sua característica de apenas apontar e identificar potenciais de melhoria na atividade que está se estudando. DEA não fornece informações suficientes de como melhorar uma certa atividade, porém ajuda a decidir se tal atividade deve ser analisada com mais detalhes (Homburg, 2001).

A afinidade entre a metodologia DEA e as práticas de benchmarking começa na fase de planejamento: tanto em um como em outro, identifica-se a organização, produto ou serviço a ser avaliado. Além disso, no benchmarking existe a procura das empresas líderes onde quer que estejam. No entanto, uma quantificação ou mensuração da empresa líder pode tornar-se impraticável. O método DEA é responsável por realizar as comparações entre empresas que se encontram em um mesmo cenário. No que se refere à coleta de dados, observa-se uma similaridade em ambos os casos, de modo que se caracterize o máximo possível a realidade.

Finalmente, o conjunto DEA - benchmarking pode apresentar uma grande vantagem no aperfeiçoamento de estudos de competição interna, já que em muitas empresas uma mesma atividade é realizada de formas distintas por diferentes unidades. As diferenças são minimizadas e as eficiências operacionais de cada unidade podem ser melhor analisadas. Esta situação pode ser observada no presente estudo de caso, já que todas as unidades de refino analisadas pertencem à mesma empresa, configurando-se em um caso típico de benchmarking interno (Silva, 1994).

\section{O Método de Análise Envoltória de Dados (DEA)}

A Análise Envoltória de Dados ou “Data Envelopment Analysis” (DEA), primeiro proposta por Charnes et al. (1978), é um método não paramétrico de análise de eficiência: ele não requer suposições a respeito da forma da fronteira de produção. DEA define a eficiência de uma unidade em relação às melhores unidades de performance observadas. Em contraste à análise de regressão, o método DEA se concentra na identificação das melhores práticas ao invés da determinação de um parâmetro médio.

DEA tem se mostrado um método adequado para a análise de eficiência de séries de unidades de produção em termos de múltiplos inputs e outputs. Tais unidades de produção são geralmente referidas como Unidades de Tomada de Decisão (DMU). DEA calcula a eficiência relativa para cada DMU comparando seus dados de inputs e outputs com todas as outras DMU's. Em adição às medidas de eficiências relativas, o método DEA provê o seguinte: 
a) Uma superfície envoltória linear que representa a fronteira de melhor prática (best pratice frontier). As unidades de melhor prática são aquelas que exibem os maiores outputs atingíveis em relação a todas as outras DMU's da população, para um dado nível de inputs, ou aquelas que utilizam o menor montante de recursos para atingir um determinado nível de outputs.

b) Uma eficiência métrica que representa o desempenho de cada DMU medida pela sua distância à fronteira.

c) Alvos específicos ou projeções de eficiência na fronteira para cada DMU ineficiente.

d) Um conjunto de referências eficientes para cada DMU definido pelas unidades eficientes mais próximas a ela. $\mathrm{O}$ conjunto de referência é observado para produzir um nível igual ou mais alto de outputs com um número igual ou inferior de inputs em relação a DMU ineficiente que está sendo comparada.

Modelos DEA são classificados com respeito ao tipo de superfície envoltória, à orientação (input ou output) e à medida de eficiência. Existem dois tipos básicos de superfícies envoltórias conhecidas como "retorno de escala constante" (CRS) e "retorno de escala variável" (VRS). Cada modelo realiza suposições implícitas a respeito de retornos de escala associados a cada tipo de superfície. O modelo CRS assume que um aumento dos outputs é proporcional ao aumento dos inputs em qualquer escala de operação. Banker et al. (1984) introduziram na literatura o modelo BCC ou VRS permitindo à tecnologia de produção exibir um aumento de retorno de escala (IRS) e uma redução de retorno de escala (DRS) assim como CRS. Os modelos DEA são também classificados como radialmente orientados a inputs, radialmente orientados a outputs ou aditivos baseados na direção da projeção das unidades ineficientes na fronteira (Paradi et al., 1997). Além disso, são dois os tipos de apresentação dos modelos DEA quanto à medida de eficiência: envelope e multiplicadores.

O resultado do método DEA é a determinação de uma superfície envelope ou fronteira Pareto-Eficiente. As DMUs que repousam sobre a superfície determinam o envelope e são consideradas eficientes, enquanto as outras são consideradas ineficientes.

A metodologia DEA calcula a eficiência relativa das unidades de tomada de decisão (DMU) com múltiplos inputs e outputs. Esta eficiência é definida como a razão do peso total dos outputs pelo peso total dos inputs. Assumindo-se que existem " $\mathrm{n}$ " DMU's para serem avaliadas, cada DMU consome quantidades variáveis de " $\mathrm{m}$ " diferentes inputs para produzir "s" diferentes outputs. Uma $\mathrm{DMU}_{\mathrm{j}}$ específica consome quantidades $\mathrm{X}_{\mathrm{j}}=\left\{\mathrm{X}_{\mathrm{ij}}\right\}$ de inputs $(\mathrm{i}=1, \ldots, \mathrm{m})$ e produz quantidades $\mathrm{Y}_{\mathrm{j}}=\left\{\mathrm{Y}_{\mathrm{rj}}\right\}$ de outputs $(\mathrm{r}=1, \ldots, \mathrm{s}) . \mathrm{X}_{\mathrm{j}}$ e $\mathrm{Y}_{\mathrm{j}}$ são as observações obtidas para cada DMU e $\mathrm{X}_{\mathrm{j}}>0$ e $\mathrm{Y}_{\mathrm{j}}>0$. Y é a matriz s X n de outputs e X é a matriz $\mathrm{m}$ x n de inputs (Paradi, 1997).

Charnes et al. (1978) desenvolveram o modelo CRS, no qual os parâmetros de entrada e saída apresentam retornos de escala proporcionais, ou seja, a duplicação de um parâmetro acarretará um aumento duas vezes maior na eficiência relacionada à unidade analisada.

Banker et al. (1984) contribuíram para que a metodologia DEA modelasse mais adequadamente a realidade com a introdução na literatura do modelo de retorno variável de escala ou VRS. Desde então, a técnica DEA tem despertado maior interesse, sendo empregada com maior intensidade em diversos campos de atuação, como por exemplo setores industriais, acadêmicos e governamentais. 
A formulação do modelo VRS é ligeiramente diferente do modelo CRS devido a existência de uma restrição adicional, cuja função é exatamente evitar a propriedade de raio ilimitado. Esta restrição obriga que o somatório dos $\lambda$ 's de todas as DMU's resulte 1, garantindo que a combinação linear para a projeção do ponto na fronteira seja convexa.

Este trabalho adota o modelo VRS na execução do método DEA, portanto, somente este modelo será descrito e analisado, tal como a seguir:

Modelo Envelope

$$
\begin{aligned}
& \text { Min h (1) } \\
& \text { s.a. } y_{r j} \leq \sum_{\mathrm{k}=1}^{\mathrm{n}} \lambda_{\mathrm{k}} \mathrm{y}_{\mathrm{rk}}, \mathrm{r}=1 . . \mathrm{s} \\
& \text { h.x } \mathrm{ij}_{\mathrm{ij}} \geq \sum_{\mathrm{k}=1}^{\mathrm{n}} \lambda_{\mathrm{k}} \mathrm{x}_{\mathrm{ik}}, \mathrm{i}=1 . \mathrm{m} \\
& \sum_{\mathrm{k}=1}^{\mathrm{n}} \lambda_{\mathrm{k}}=1 \\
& \mathrm{~h} \text { irr, } \lambda_{\mathrm{k}} \geq 0, \mathrm{k}=1 . . \mathrm{n}
\end{aligned}
$$

\section{Modelo Multiplicadores}

$$
\begin{aligned}
& \operatorname{Max} \sum_{\mathrm{r}=1}^{\mathrm{s}} \mathrm{y}_{\mathrm{rj}} \mathrm{u}_{\mathrm{r}}+\mathrm{u}^{*}(6) \\
& \text { s.a. } \sum_{\mathrm{i}=1}^{\mathrm{m}} \mathrm{x}_{\mathrm{ij}} \mathrm{v}_{\mathrm{i}}=1 \\
& \sum_{\mathrm{i}=1}^{\mathrm{s}} \mathrm{y}_{\mathrm{rk}} \mathrm{u}_{\mathrm{r}}+\mathrm{u} *-\sum_{\mathrm{i}=1}^{\mathrm{m}} \mathrm{x}_{\mathrm{ik}} \mathrm{v}_{\mathrm{i}} \leq 0, \mathrm{k}=1 . . \mathrm{n} \\
& \mathrm{v}_{\mathrm{i}} \geq 0, \mathrm{u}_{\mathrm{r}} \geq 0, \mathrm{u} * \operatorname{irr}
\end{aligned}
$$

O modelo do envelope apresenta uma ou mais variáveis ligadas ao aumento dos outputs ou redução dos inputs para que a unidade em questão atinja a fronteira definida pelas DMU's eficientes. Há também uma variável que auxilia as restrições do modelo concedendo pesos a cada unidade estudada, no sentido de garantir que as projeções dos parâmetros de entrada e saída do sistema se encontrem dentro da região definida pela combinação linear convexa desses pesos pelo parâmetro relacionado à unidade em questão.

Efetuando-se uma análise sobre o modelo do envelope exposto acima, observa-se que a função objetivo (1) visa minimizar a variável $h$, que representa a eficiência da unidade em relação à manipulação de seus insumos (inputs). Segundo Lins \& Meza (1999), essa variável representa o menor valor (sempre menor que 1), tal que, multiplicado pelo vetor de insumos desta DMU, resulta em uma redução equiproporcional destes insumos. Busca-se a máxima redução equiproporcional (o mínimo h), tal que possamos garantir que a DMU, operando com esta nova combinação de insumos, ainda pertença ao conjunto de possibilidades de produção definido por seus inputs e outputs. Para isto, o vetor de inputs e outputs (hx,y) deve satisfazer às restrições (2) e (3). Esta última não permite que a unidade em questão se situe fora da região que expressa o conjunto de possibilidades de produção do sistema (definida pela combinação linear do parâmetro $\lambda$ pelo input das demais DMU's). Na restrição (2), a variável $\lambda$ garante que o output da DMU j esteja contido na combinação linear daquele output pelos $\lambda$ 's das outras DMU's. A restrição (4) assegura que a combinação linear para a projeção do ponto na fronteira seja convexa.

O modelo dos multiplicadores atribui pesos que são multiplicados aos parâmetros de entrada (7) e saída (6) do sistema, de forma que a eficiência de uma DMU seja calculada através da razão entre o produto dos seus outputs por seus pesos correspondentes e o produto dos seus inputs por seus pesos correspondentes. Esta razão expressa a eficiência da DMU analisada e as restrições do problema trabalham para garantir que não haja nenhuma DMU com eficiência superior a 100\% (8). Os modelos dos multiplicadores e do envelope compõem um par primal-dual (Calôba, 2003). 


\section{A Modelagem do Problema}

O presente estudo de caso, como já fora mencionado, objetiva analisar a eficiência operacional de dez refinarias de uma empresa de petróleo multinacional, de forma que as melhores práticas sejam detectadas pelo método DEA. O objetivo é a criação de um conjunto de informações quantitativas, obtidas pela execução do método, que poderão ser utilizadas como insumo para possíveis mudanças organizacionais ou qualitativas (Vasconcellos, 2003).

Para que a metodologia DEA seja empregada em um sistema, um modelo representativo do problema em questão deve ser criado. Este modelo baseia-se em parâmetros de entrada e saída característicos do sistema a ser analisado pelo método. Os parâmetros de entrada (inputs) são os recursos consumidos pelo sistema em questão para produzir um determinado resultado ou saída (output). Este é o ponto de partida para a aplicação da técnica DEA. A eficiência das unidades, quanto à manipulação dos recursos na obtenção dos seus resultados, é calculada em função das informações determinadas na modelagem do problema. O modelo DEA permite que se trabalhe com variáveis multidisciplinares e esta característica foi determinante na seleção.

As variáveis selecionadas para o modelo constituem-se em um input $\left(\mathrm{X}_{1}\right)$ e três outputs $\left(\mathrm{Y}_{1}, \mathrm{Y}_{2}\right.$ e $\left.\mathrm{Y}_{3}\right)$. Estes parâmetros são representativos da atividade de refino e foram coletados na literatura. As características e os critérios de seleção das variáveis estão detalhadamente descritos nas linhas subseqüentes:

$X_{I}$ - Capacidade Ociosa: é o percentual projetado da refinaria que não está efetivamente sendo utilizado. É medido por um fator denominado fator de utilização (f.u.), que é a razão da capacidade efetiva pela capacidade de projeto. A capacidade ociosa é calculada pela expressão $(1-$ f.u.). Quanto mais próximo de 0 , maior a utilização da capacidade projetada e conseqüentemente, um volume maior de óleo é processado, gerando mais produto. Este parâmetro foi adotado como o input do modelo, pois ele expressa o potencial de recursos disponíveis pela unidade (utilidades, energia, capacidade de processar óleo bruto, etc.) que não são consumidos e que deixam de ser convertidos em produtos. Minimizar o parâmetro "capacidade ociosa" significa afirmar que se pretende aumentar a capacidade de processamento de óleo pela unidade e favorecer a geração de produtos.

$Y_{I}$ - Percentual de derivados leves: expressa a fração de derivados leves que é convertida através do processamento do petróleo nas refinarias. Este parâmetro é interessante, visto que os derivados leves possuem valor agregado mais elevado. Por esse motivo, eles contribuem mais efetivamente na receitas das refinarias.

$Y_{2}-$ Receita $/ \mathrm{m}^{3}$ de óleo processado: expressa o valor anual (em dólares) arrecadado pela refinaria por $\mathrm{m}^{3}$ de óleo processado. Esta quantia é calculada a partir dos preços de realização (sem os impostos) das unidades. Este output reduz o efeito de escala das refinarias, colocando no mesmo patamar de comparação todas as DMU's, independente do valor absoluto da receita obtida.

$Y_{3}$ - Produção de Gasolina A / $100 \mathrm{~m}^{3}$ de óleo processado: este fator expressa o volume produzido de gasolina A por $100 \mathrm{~m}^{3}$ de óleo processado. O critério para a seleção desta variável baseou-se no fato da gasolina A apresentar um alto valor agregado, uma grande significância para indústria e para sociedade, além de ser alvo de produção por parte de todas as refinarias, dado o seu enorme consumo em todo o país (principalmente nos grandes centros urbanos). A gasolina A diferencia-se da gasolina $\mathrm{C}$ vendida pelas distribuidoras nos postos pela ausência dos $24 \%$ (em base volumétrica) de álcool anidro combustível (AEAC). 
O modelo gerado pode ser visualizado segundo o seguinte esquema (Figura 1):

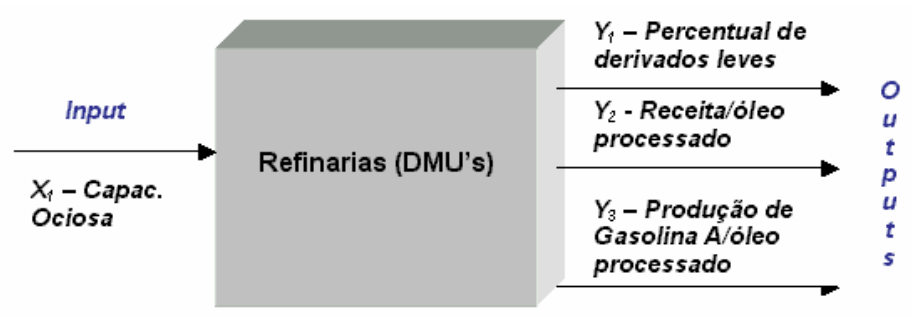

Figura 1 - Ilustração da modelagem do problema.

A tabela abaixo aloca os valores dos inputs e outputs do modelo proposto, por unidade de refino. Estes valores são executados pelo método DEA, que calcula a eficiência relativa de cada DMU do sistema.

Tabela 1 - Dados do problema.

\begin{tabular}{|c|c|c|c|c|}
\hline & Input & \multicolumn{3}{|c|}{ Outputs } \\
\hline DMU's & $\begin{array}{c}\text { Capacidade } \\
\text { Ociosa }\end{array}$ & \% Derivados Leves & $\begin{array}{c}\text { Receita/Óleo } \\
\text { Processado } \\
\left(\mathbf{U S \$} / \mathbf{m}^{3}\right)\end{array}$ & $\begin{array}{c}\text { Produção de } \\
\text { Gasolina A/ } 100 \mathrm{~m}^{3} \\
\text { de Óleo Processado }\end{array}$ \\
\hline R1 & 0,09 & 0,99 & 179,25 & 13,0 \\
\hline $\mathbf{R 2}$ & 0,22 & 0,77 & 181,58 & 9,0 \\
\hline R3 & 0,20 & 0,87 & 173,58 & 19,0 \\
\hline R4 & 0,43 & 0,90 & 177,00 & 18,0 \\
\hline R5 & 0,16 & 0,83 & 184,86 & 22,0 \\
\hline R6 & 0,01 & 0,87 & 222,19 & 17,0 \\
\hline $\mathbf{R 7}$ & 0,08 & 0,90 & 211,60 & 26,0 \\
\hline R8 & 0,19 & 0,99 & 203,30 & 35,0 \\
\hline R9 & 0,05 & 0,77 & 166,80 & 10,0 \\
\hline $\mathbf{R 1 0}$ & 0,01 & 0,85 & 178,07 & 20,0 \\
\hline
\end{tabular}

Após as etapas de modelagem do problema e coleta de dados, têm-se todas as condições para a execução do modelo matemático. Porém, para que isso aconteça, é necessário estruturar os dados na forma matemática exigida pelo método DEA, de acordo com as características do sistema.

No estudo de caso atual, escolheu-se o modelo com retorno de escala variável (VRS), no formato multiplicadores, sob a ótica dos outputs e com restrição aos pesos. A ótica de maximização de outputs provém da predominância deste tipo de parâmetro no modelo e o retorno variável de escala é explicado pela própria característica dos dados coletados, onde não se percebe uma relação uniforme entre as escalas dos mesmos. Por este motivo, segundo Banker et al. (1984), o modelo VRS tem a característica de se aproximar mais à realidade que o modelo CRS. A adoção das restrições aos pesos justifica-se pelo fato de se desejar evitar a ocorrência de DMU's Pareto-Ineficientes na execução do método. Desta maneira não se permitem que alguns parâmetros recebam peso zero, o que poderia mascarar os resultados. 
A modelagem do problema, em seu formato matemático, pode ser visualizada através da seguinte formulação:

\section{DMU R-1}

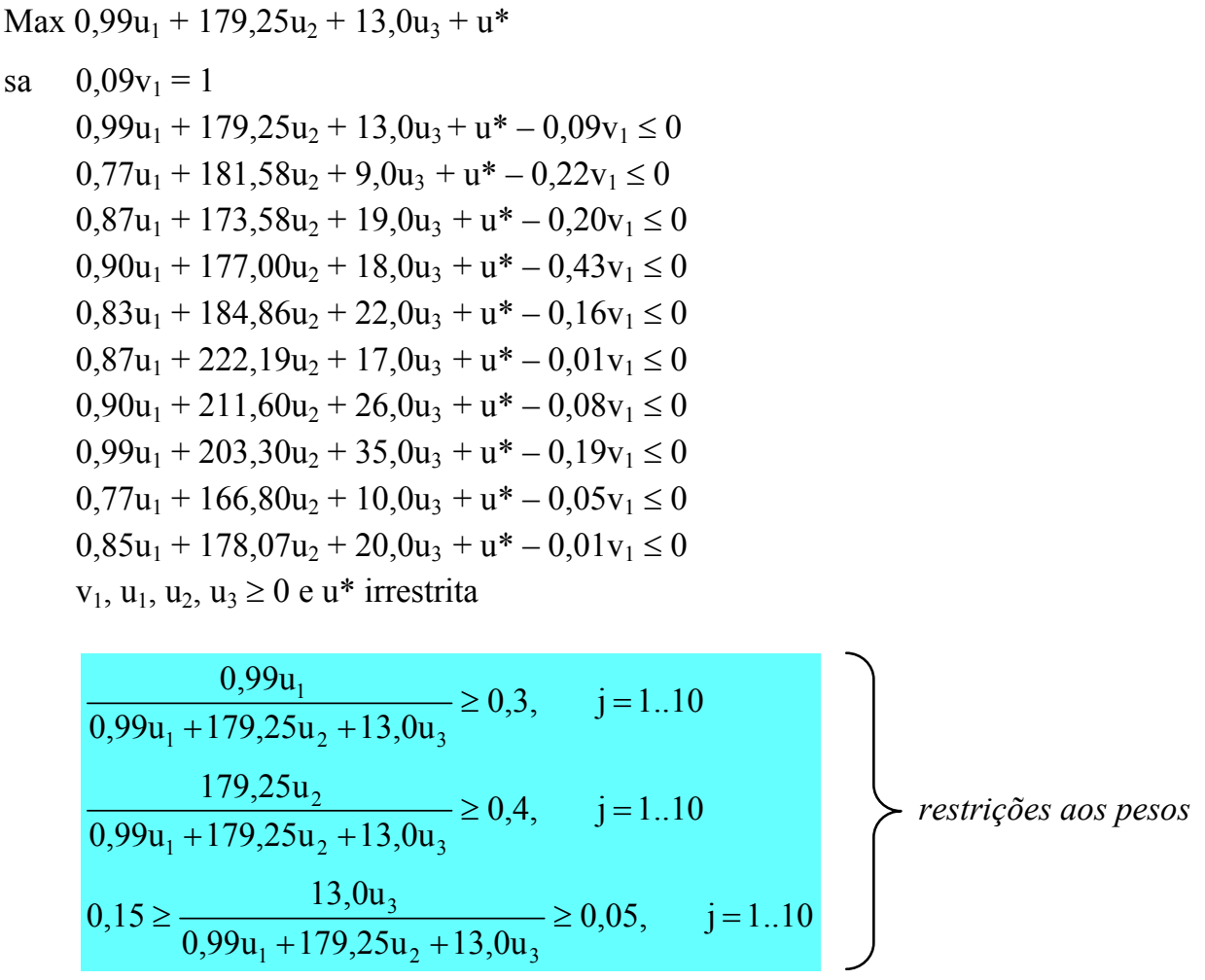

Vale destacar que a formulação acima é referente apenas à DMU R-1. Para a formulação dos modelos das outras unidades, deve-se alterar a função objetivo e a primeira restrição do modelo de programação linear da DMU em questão, mantendo-se inalteradas as demais restrições.

Foram utilizadas restrições aos pesos em adição às do modelo DEA tradicional, conforme destacado na formulação acima. Um modelo DEA sem restrições aos pesos pode atribuir peso zero a algum parâmetro de uma determinada unidade cujo desempenho é inferior às demais unidades envolvidas. Este fato constitui um caso de ocorrência das chamadas DMU's Pareto-Ineficientes, que são aquelas situadas na fronteira eficiente, mas que não correspondem à premissa básica de eficiência definida pelo método DEA, que é a de utilizar um mínimo nível de recurso para atingir um dado nível de resultado, ou alcançar um máximo nível de desempenho em relação às saídas, utilizando um dado nível de entradas. Uma DMU Pareto-Ineficiente sempre pode apresentar uma melhora de desempenho se comparada às DMU's eficientes, seja ela por redução de inputs ou aumento de outputs. Em outras palavras, uma DMU eficiente pode ser referência para DMU's ineficientes e Pareto-Ineficientes, caso estas ocorram. Para que isso seja evitado, foram empregados os seguintes limites de atuação para cada output, que deram origem às restrições aos pesos utilizadas na formulação: 
Tabela 2 - Restrições aos pesos.

\begin{tabular}{|l|c|}
\hline \multicolumn{1}{|c|}{ Outputs } & Limites de contribuição \\
\hline $\mathrm{Y}_{1}-$ Percentual de Derivados Leves & 30 a $100 \%$ \\
\hline $\mathrm{Y}_{2}-$ Receita/Óleo Processado & 40 a $100 \%$ \\
\hline $\mathrm{Y}_{3}-$ Produção de Gasolina A/Óleo Processado & 5 a $15 \%$ \\
\hline
\end{tabular}

Os percentuais foram definidos através de consultas a especialistas da área, tendo prevalecido a experiência prática desses profissionais. Como o modelo visa a maximização dos outputs, os inputs não receberam restrições aos pesos. Vale ressaltar que estas restrições são caracterizadas por não atuarem sobre o valor dos pesos atribuídos aos parâmetros, e sim sobre os chamados "outputs virtuais", que são o produto dos pesos pelos parâmetros de saída do modelo. É importante ressaltar que a contribuição de um output $\mathrm{r}$ em relação à DMU j é $\mathrm{u}_{\mathrm{r}} \mathrm{y}_{\mathrm{rj}}$.

\section{Resultados e Análises}

Os resultados alocados nas Tabelas 3 e 4 foram gerados através da execução do software Frontier Analyst, que forneceu os alvos de desempenho e os potenciais de melhoria.

Tabela 3 - Eficiências.

\begin{tabular}{|l|c|}
\hline \multicolumn{1}{|c|}{ DMU's } & Eficiência (\%) \\
\hline R-8 & 100,00 \\
\hline R-6 & 100,00 \\
\hline R-7 & 99,76 \\
\hline R-1 & 92,06 \\
\hline R-10 & 91,98 \\
\hline
\end{tabular}

\begin{tabular}{|l|c|}
\hline \multicolumn{1}{|c|}{ DMU's } & Eficiência (\%) \\
\hline R-5 & 86,78 \\
\hline R-4 & 86,08 \\
\hline R-3 & 84,18 \\
\hline R-9 & 78,71 \\
\hline R-2 & 76,46 \\
\hline
\end{tabular}

Tabela 4 - Alvos de desempenho e potenciais de melhoria (\%).

\begin{tabular}{|l|c|c|c|c|c|c|c|c|c|}
\cline { 2 - 10 } \multicolumn{1}{c|}{} & \multicolumn{3}{c|}{ DMU R-8 } & \multicolumn{3}{c|}{ DMU R-6 } & \multicolumn{3}{c|}{ DMU R-7 } \\
\hline \multicolumn{1}{|c|}{ Parâmetros } & Atual & Alvo & $\begin{array}{c}\text { Pot. } \\
\text { Melh. }\end{array}$ & Atual & Alvo & $\begin{array}{c}\text { Pot. } \\
\text { Melh. }\end{array}$ & Atual & Alvo & $\begin{array}{c}\text { Pot. } \\
\text { Melh. }\end{array}$ \\
\hline $\begin{array}{l}\text { Capacidade } \\
\text { Ociosa }\end{array}$ & 0,19 & 0,19 & 0 & 0,01 & 0,01 & 0 & 0,08 & 0,08 & 0 \\
\hline $\begin{array}{l}\text { \% Derivados } \\
\text { Leves }\end{array}$ & 0,99 & 0,99 & 0 & 0,87 & 0,87 & 0 & 0,9 & 0,92 & 1,85 \\
\hline $\begin{array}{l}\text { Receita / } \\
\text { Óleo Proc. }\end{array}$ & 203,3 & 203,3 & 0 & 222,19 & 222,19 & 0 & 211,6 & 214,84 & 1,53 \\
\hline $\begin{array}{l}\text { Gasolina A/ } \\
\text { Óleo Proc. }\end{array}$ & 35 & 35 & 0 & 17 & 17 & 0 & 26 & 24 & $-7,69$ \\
\hline
\end{tabular}




\begin{tabular}{|l|c|c|c|c|c|c|c|c|c|}
\cline { 2 - 10 } \multicolumn{1}{c|}{} & \multicolumn{3}{c|}{ DMU R-1 } & \multicolumn{3}{c|}{ DMU R-10 } & \multicolumn{3}{c|}{ DMU R-5 } \\
\hline $\begin{array}{l}\text { Parâmetros } \\
\text { Capacidade } \\
\text { Ociosa }\end{array}$ & Atual & Alvo & $\begin{array}{c}\text { Pot. } \\
\text { Melh. }\end{array}$ & Atual & Alvo & $\begin{array}{c}\text { Pot. } \\
\text { Melh. }\end{array}$ & Atual & Alvo & $\begin{array}{c}\text { Pot. } \\
\text { Melh. }\end{array}$ \\
\hline $\begin{array}{l}\text { \% Derivados } \\
\text { Leves }\end{array}$ & 0,99 & 0,92 & $-6,73$ & 0,99 & 0,87 & 2,35 & 0,83 & 0,97 & 16,87 \\
\hline $\begin{array}{l}\text { Receita / } \\
\text { Óleo Proc. }\end{array}$ & 179,25 & 213,79 & 19,27 & 179,25 & 222,19 & 24,78 & 184,86 & 206,45 & 11,68 \\
\hline $\begin{array}{l}\text { Gasolina A/ } \\
\text { Óleo Proc. }\end{array}$ & 13 & 25 & 92,31 & 13 & 17 & -15 & 22 & 32 & 45,45 \\
\hline
\end{tabular}

\begin{tabular}{|l|c|c|c|c|c|c|c|c|c|c|c|c|}
\cline { 2 - 12 } \multicolumn{1}{c|}{} & \multicolumn{3}{c|}{ DMU R-4 } & \multicolumn{3}{c|}{ DMU R-3 } & \multicolumn{3}{c|}{ DMU R-9 } & \multicolumn{3}{c|}{ DMU R-2 } \\
\hline Parâmetros & Atual & Alvo & $\begin{array}{c}\text { Pot. } \\
\text { Melh. }\end{array}$ & Atual & Alvo & $\begin{array}{c}\text { Pot. } \\
\text { Melh. }\end{array}$ & Atual & Alvo & $\begin{array}{c}\text { Pot. } \\
\text { Melh. }\end{array}$ & Atual & Alvo & $\begin{array}{c}\text { Pot. } \\
\text { Melh. }\end{array}$ \\
\hline $\begin{array}{l}\text { Capacidade } \\
\text { Ociosa }\end{array}$ & 0,43 & 0,19 & $-55,81$ & 0,2 & 0,19 & -5 & 0,05 & 0,05 & 0 & 0,22 & 0,19 & $-13,64$ \\
\hline $\begin{array}{l}\text { \% Derivados } \\
\text { Leves }\end{array}$ & 0,9 & 0,99 & 10 & 0,87 & 0,99 & 13,79 & 0,77 & 0,9 & 16,45 & 0,77 & 0,99 & 28,57 \\
$\begin{array}{l}\text { Receita / } \\
\text { Óleo Proc. }\end{array}$ & 177 & 203,3 & 14,86 & 173,58 & 203,3 & 17,12 & 166,8 & 217,99 & 30,69 & 181,58 & 203,3 & 11,96 \\
\hline $\begin{array}{l}\text { Gasolina A/ } \\
\text { Óleo Proc. }\end{array}$ & 18 & 35 & 94,44 & 19 & 35 & 84,21 & 10 & 21 & 110 & 9 & 35 & 288,89 \\
\hline
\end{tabular}

Tabela 5 - Contribuição dos parâmetros do modelo para as DMU's ineficientes.

\begin{tabular}{|l|c|c|c|c|c|c|c|c|c|c|c|c|}
\hline & \multicolumn{10}{|c|}{ Contribuição \% dos Parâmetros - DMU's de Referência por DMU Ineficiente Analisada } \\
\hline \multirow{2}{*}{ Parâmetros } & \multicolumn{2}{|c|}{ R-1 } & R-2 & R-3 & R-4 & \multicolumn{2}{|c|}{ R-5 } & \multicolumn{2}{|c|}{ R-7 } & \multicolumn{2}{|c|}{ R-9 } & R-10 \\
\cline { 2 - 13 } & R-6 & R-8 & R-8 & R-8 & R-8 & R-6 & R-8 & R-6 & R-8 & R-6 & R-8 & R-6 \\
\hline $\begin{array}{l}\text { Capacidade } \\
\text { Ociosa }\end{array}$ & 6,17 & 93,83 & 100 & 100 & 100 & 1,04 & 98,96 & 7,64 & 92,36 & 15,56 & 84,44 & 100 \\
\hline $\begin{array}{l}\text { \% Derivados } \\
\text { Leves }\end{array}$ & 52,35 & 47,65 & 100 & 100 & 100 & 14,95 & 85,05 & 58,00 & 42,00 & 75,46 & 24,54 & 100 \\
\hline $\begin{array}{l}\text { Receita / } \\
\text { Óleo Proc. }\end{array}$ & 57,74 & 42,26 & 100 & 100 & 100 & 17,94 & 82,06 & 63,20 & 36,80 & 79,28 & 20,72 & 100 \\
\hline $\begin{array}{l}\text { Gasolina A/ } \\
\text { Óleo Proc. }\end{array}$ & 37,78 & 62,22 & 100 & 100 & 100 & 8,85 & 91,15 & 43,29 & 56,71 & 62,96 & 37,04 & 100 \\
\hline
\end{tabular}

A Tabela 3 revela as unidades de refino mais eficientes da empresa estudada, com base nos parâmetros do modelo proposto: R-6 e R-8. Estas representam o "estado da arte" no que se refere ao consumo dos recursos e geração dos resultados alcançados. Dentro do contexto criado, essas DMU's são consideradas detentoras das melhores práticas operacionais e devem ser tomadas como referência no estudo de benchmarking. Na interpretação gráfica do método DEA, as referidas DMU's constituem a chamada fronteira "Pareto-Eficiente" ou fronteira de melhor prática (best pratice frontier).

O método quantifica a contribuição das DMU's de referência, de acordo com a Tabela 5. A DMU R-8 contribui mais efetivamente como referência para as unidades ineficientes. Seus parâmetros são utilizados como "espelho" por quase todas as DMU's ineficientes, sendo que 
em 3 delas (R-2, R-3 e R-4) isso ocorre integralmente. Já a DMU R-6 contribui como referência parcial para 4 DMU's (R-1, R-5, R-7 e R-9) e integral para a DMU R-10.

A Tabela 4 exibe os valores de melhoria potencial para os inputs e outputs das DMU's. A coluna "Valor Atual" contém os valores originais dos parâmetros do modelo. A coluna "Alvo" refere-se aos valores dos parâmetros correspondentes das DMU's de referência de cada unidade em particular. O conjunto de referência é observado para produzir um nível igual ou mais alto de outputs com um número igual ou inferior de inputs em relação a DMU ineficiente que está sendo comparada.

Sob a ótica da filosofia benchmarking, os resultados apresentados nas colunas "Alvo" e "Melhoria Potencial", expressam quantitativamente toda a busca pela excelência operacional daquelas unidades que não detém as melhores práticas referentes à manipulação dos parâmetros do modelo proposto. Esses valores propiciam a dimensão exata dos esforços a serem despendidos no caminho da melhoria contínua rumo a excelência. Trata-se de dados quantitativos, porém são informações preciosas para a implementação das mudanças qualitativas visando novos resultados.

A Figura 2 exibe um exemplo de fronteira de eficiência formada pelas DMU's eficientes do modelo através da plotagem do input "Capacidade Ociosa" pelo output "Produção de Gasolina A/100 $\mathrm{m}^{3}$ de Óleo Processado". Observa-se que as unidades menos eficientes são aquelas que se encontram mais afastadas da fronteira. Outro fato interessante que deve ser notado em relação à disposição dos pontos no gráfico, é que as DMU's que se situam à esquerda da DMU R-8 são aquelas que têm como referência uma combinação entre as DMU's eficientes R-6 e R-8.

Já as que se localizam à direita da DMU R-8 (R-2, R-3 e R-4), são aquelas que são influenciadas integralmente por esta unidade eficiente. Isto se explica através da projeção do ponto representativo da unidade no gráfico na fronteira. A distância vertical entre cada ponto e a fronteira representa o percentual necessário de melhoria que a unidade terá que conseguir para atingir o patamar de eficiência. Percebe-se que, como se trata de um modelo de maximização de outputs, este percentual é positivo, ou seja, a DMU deve aumentar a geração de outputs para alcançar a eficiência operacional.

Abre-se uma exceção para as DMU's R-7 e R-10, que em um fato pouco comum, ultrapassaram o patamar eficiente expresso pela fronteira e, para alcançá-la, devem reduzir suas produções em $7,69 \%$ e $15 \%$ respectivamente. Isto ocorre, em primeiro lugar, devido ao bom desempenho apresentado por essas DMU's em relação aos parâmetros em questão. Quanto às suas posições acima da fronteira eficiente, pode-se afirmar que o emprego das restrições aos pesos evitou que os pontos referentes à essas DMU's se localizassem sobre o segmento eficiente formado pelas DMU's R-6 e R-8, o que configuraria um caso de ocorrência das chamadas DMU's Pareto-Ineficientes, ou seja, aquelas que se localizam sobre a fronteira, mas que são unidades ineficientes.

A DMU que se encontra mais próxima à fronteira (R-5) é a que deve investir menos no aumento da produção de gasolina A $(45,45 \%)$. Já a DMU R-2 apresenta o pior desempenho de todas as unidades, precisando aumentar sua produção de gasolina A em $288,89 \%$. Estes valores podem ser considerados elevados e comprovam que o output "Produção de Gasolina A" é o que apresenta os resultados menos alentadores para as unidades. Este fato é bastante nítido no gráfico, já que os pontos estão muito afastados da fronteira. Há, portanto, um gap gritante entre as unidades eficientes e as demais e nada como um estudo de benchmarking para investigar como essa diferença pode ser diminuída. 


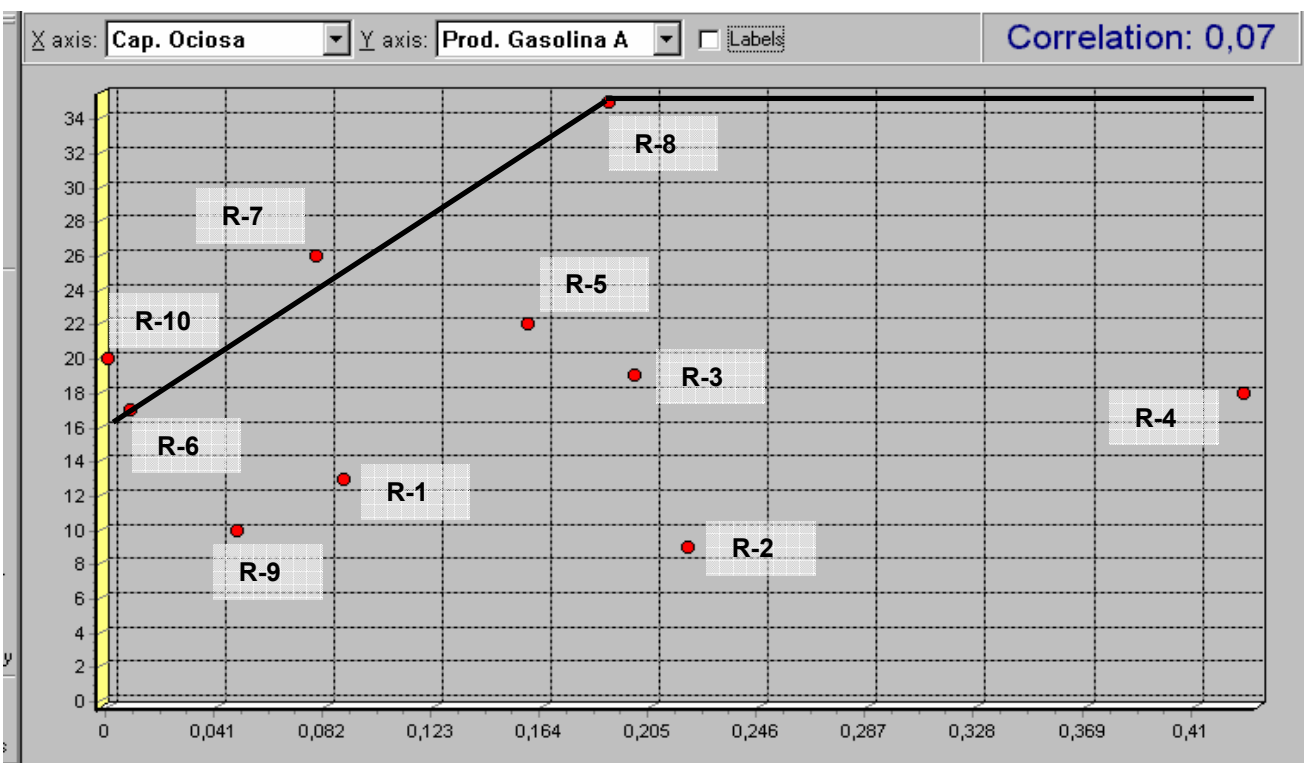

Figura 2 - Capacidade Ociosa ("X") vs Produção de Gasolina A/100m³ de Óleo Processado ("Y”) (extraído do software Frontier Analyst).

\section{Plano de Melhorias}

A proposta do presente estudo não vai além da elaboração de um plano de melhorias. Conforme o que fora mencionado anteriormente, o tipo de processo de benchmarking adotado no trabalho é quantitativo, o que não impede absolutamente que se formule um planejamento, de forma a identificar novos caminhos ou mudanças a serem seguidos com base nas melhores práticas detectadas pelo método DEA. O estudo não entra no mérito da execução desses planos de melhoria, apenas aponta possíveis focos de mudanças.

\section{Redução do Input "Capacidade Ociosa"}

Tabela 6 - Medidas qualitativas para redução do input "Capacidade Ociosa".

\begin{tabular}{|c|l|l|l|}
\hline Medida & Responsável & Tempo & Unidades-Alvo \\
\hline Aumentar a carga de refino & Corporativo & Curto prazo & Todas as ineficientes, exceto R-10 \\
\hline
\end{tabular}

O plano de melhoria deve prever uma única ação em relação ao input "Capacidade Ociosa", que é o aumento da carga de refino (de acordo com a Tabela 6). Esta ação permite que a unidade opere em um patamar superior de alimentação. A produção aumenta, os outputs do modelo são favorecidos e a unidade torna-se mais competitiva. Não há necessidade de mudanças na capacidade projetada. O prazo para implementação do ajuste é curto. As conseqüências desse ajuste no decorrer no processo devem ser avaliadas, tanto no âmbito financeiro, quanto no operacional. Este plano deve ser acompanhado de um estudo com o intuito de verificar se o aumento da produção pode ser absorvido pelo mercado. 
O processo de benchmarking deve ser capaz de diagnosticar e apontar possibilidades de melhoria de desempenho para cada refinaria analisada. Para que o processo seja executado com êxito, o Plano de Melhorias proposto em função dos resultados obtidos pelo método DEA, deve ser capaz de se adaptar aos diferentes esquemas de refino e atender as necessidades específicas de melhoria de cada unidade. Caso contrário, o processo teria apenas um caráter genérico com probabilidade de contemplar medidas ineficazes e incapazes de gerar um aumento de performance em função de alguma particularidade da unidade.

No que se refere à diminuição do input "Capacidade Ociosa", o seguinte aspecto foi levado em consideração: se a unidade de refino tem capacidade projetada suficiente para suportar um aumento de carga. Sob este ponto de vista, todas as unidades ineficientes se enquadram no plano. As únicas refinarias que não teriam condições de se adequar à medida propostas seriam a R-6 e a R-10, por já operarem em patamar limite em termos de capacidade, de acordo com os dados da Tabela 2. Mas estas duas unidades não são levadas em consideração pelo plano, pois o Quadro 4 mostra que estas unidades trabalham bem o parâmetro em questão (a DMU R-6, inclusive, é eficiente), já que nos 2 casos não há potencial de melhoria detectado pelo método DEA.

\section{Aumento do Output "Percentual de Derivados Leves"}

Tabela 7 - Medidas qualitativas para aumento do output "Percentual de Derivados Leves".

\begin{tabular}{|l|l|l|l|}
\hline \multicolumn{1}{|c|}{ Medida } & Responsável & \multicolumn{1}{c|}{ Tempo } & \multicolumn{1}{c|}{ Unidades-Alvo } \\
\hline $\begin{array}{l}\text { Otimizar a eficiência da } \\
\text { operação de destilação }\end{array}$ & Projeto & Médio prazo & Todas as ineficientes \\
\hline $\begin{array}{l}\text { Adaptar a Unidade para o } \\
\text { conceito "fundo de barril” }\end{array}$ & Projeto & Longo prazo & R-2, R-3, R-4, R-9 e R-10 \\
\hline Investir em novos processos & Corporativo & Longo prazo & Todas as ineficientes \\
\hline
\end{tabular}

As ações relativas ao output "Percentual de Derivados Leves" contempladas no Plano de Melhoria e que são observadas na Tabela 7, têm uma relação estreita entre si e devem estar inteiramente integradas. A primeira proposta visa buscar a maior eficiência do processo de destilação atmosférica, que é a primeira etapa de separação entre leves e pesados. Os processos subseqüentes são totalmente dependentes da etapa de destilação. Portanto, cabe ao setor de projeto dimensionar as torres fracionadoras (aumentando o número de estágios de transferência de calor e massa, por exemplo), de forma que o produto apresente maior composição de leves. Todas as unidades consideradas ineficientes no estudo apresentam em seus esquemas de refino unidades de destilação. Logo, a medida mencionada é factível em todas elas, sem exceção.

A proposta de adaptação das unidades para o conceito "fundo de barril", conseqüência do processamento de petróleo denso pelas refinarias, principalmente aquelas que não têm o objetivo de produzir grandes volumes de derivados pesados para o mercado. Estes processos visam converter as frações mais pesadas provenientes da destilação a vácuo em frações mais leves e de maior valor agregado. Os processos Coqueamento Retardado e Craqueamento Catalítico Fluido (FCC - Fluid Catalytic Cracking) enquadram-se neste objetivo. Os investimentos em novos equipamentos e instalações são altos e o prazo para implementação destas ações é longo. Como todas as refinarias apresentam em suas configurações o processo 
FCC, o critério para a definição das unidades-alvo onde esta medida é factível é direcionado na existência do processo Coqueamento Retardado. As unidades R-2, R-3, R-4, R-9 e R-10 são os alvos de melhoria da medida em questão, por ainda não disporem deste processo em seus esquemas de refino.

\section{Aumento do Output "Receita/m $\mathbf{m}^{3}$ de Óleo Processado"}

Tabela 8 - Medidas qualitativas para aumento do output "Receita/ $\mathrm{m}^{3}$ de Óleo Processado".

\begin{tabular}{|l|l|l|l|}
\hline \multicolumn{1}{|c|}{ Medida } & Responsável & \multicolumn{1}{c|}{ Tempo } & \multicolumn{1}{c|}{ Unidades-Alvo } \\
\hline $\begin{array}{l}\text { Aumento da produção de } \\
\text { derivados leves (ver item 5.8.2) }\end{array}$ & Corporativo & $\begin{array}{l}\text { Médio / } \\
\text { Longo prazo }\end{array}$ & Todas as ineficientes, exceto R-1 \\
\hline Otimizar o processo & Projeto & Médio prazo & Todas as ineficientes \\
\hline
\end{tabular}

O Plano de Melhoria em relação ao parâmetro "Receita $/ \mathrm{m}^{3}$ de Óleo Processado" contempla o aumento da produção de derivados leves como uma das suas principais ações, de acordo com a Tabela 8. Isto pode ser explicado pelo fato destes derivados possuírem maior valor agregado em comparação aos pesados, contribuindo mais efetivamente para as receitas das unidades de refino. Neste caso as medidas adotadas na Tabela 7 também podem ser aplicadas, com uma única ressalva que diz respeito à R-1: esta é a única unidade que não se enquadra nas medidas que visam aumentar a produção de derivados leves já que, em uma exceção à regra, esta produção deve ser reduzida para que se atinja a eficiência operacional.

A segunda medida do plano alega que o processo deve ser otimizado, de forma que se consiga gerar o maior volume possível de derivados por cada $\mathrm{m}^{3}$ de óleo processado e que, conseqüentemente, seja atingido o máximo patamar de receita. Neste caso todas as unidades consideradas ineficientes podem ser alvos desta medida.

\section{Aumento do Output "Produção de Gasolina A/100 m³ de Óleo Processado"}

Tabela 9 - Medidas qualitativas para aumento do output "Produção de Gasolina A/ $100 \mathrm{~m}^{3}$ de Óleo Processado".

\begin{tabular}{|l|l|l|l|}
\hline \multicolumn{1}{|c|}{ Medida } & Responsável & \multicolumn{1}{c|}{ Tempo } & \multicolumn{1}{c|}{ Unidades-Alvo } \\
\hline $\begin{array}{l}\text { Adaptar a Unidade para o } \\
\text { conceito "fundo de barril" }\end{array}$ & Projeto & Longo prazo & R-2, R-3, R-4, R-9 e R-10 \\
\hline $\begin{array}{l}\text { Ajustar o processo FCC para } \\
\text { maior produção de gasolina }\end{array}$ & Projeto & Curto prazo & Todas as ineficientes \\
\hline Investir no processo de HDT & Corporativo & Médio prazo & R-2 e R-4 \\
\hline
\end{tabular}

Observa-se, através da Tabela 9, que a medida de adaptação das unidades para o conceito "fundo de barril" também é importante para favorecer o aumento da produção de gasolina A por cada $100 \mathrm{~m}^{3}$ de óleo processado. A produção de gasolina A é incrementada porque as frações pesadas provenientes da destilação a vácuo são convertidas em leves através dos processos Coqueamento Retardado e FCC. As unidades-alvo dessa medida e os motivos que contribuíram para essa definição são os mesmos mencionados na Tabela 7. 
Nos processos "fundo de barril" são produzidos, junto com a gasolina, outros tipos de derivados leves tais como GLP, nafta, querosene e diesel. Para favorecer a produção de gasolina A em relação aos demais derivados, deve-se efetuar um ajuste nas condições operacionais do reator de FCC, já que este processo é um grande produtor de gasolina. Todas as unidades consideradas ineficientes neste estudo dispõem do processo FCC em seus esquemas de refino. Logo, a referida medida é aplicável sem restrições em todas estas unidades.

Para que a nafta produzida nas unidades de Coqueamento Retardado seja aproveitada para favorecer a produção de gasolina $\mathrm{A}$, há a necessidade de inserir no esquema de refino, as unidades de hidrotratamento (HDT), pois estas naftas são extremamente olefínicas. Com a inclusão do HDT no esquema de refino, o volume de gasolina A pode ser incrementado através do aproveitamento da nafta DD (da destilação direta) como carga do processo. A nafta DD suaviza as condições operacionais e aumenta o tempo de campanha das unidades de HDT de nafta de coque. As unidades-alvo desta medida são as seguintes: R-4, que é a única ineficiente que não apresenta o processo HDT em seu esquema de refino e R-2, cuja unidade de HDT precisa ser adaptada para produção de combustíveis, já que seu foco é a produção de lubrificantes.

O presente plano de ações considera apenas aspectos operacionais no que diz respeito ao aumento da produção do output em questão. Não se discute se tais medidas seriam viáveis sob o ponto de vista do mercado, porém recomenda-se um tipo de estudo complementar diferenciado, com indicadores específicos.

\section{Conclusões}

Este trabalho sugeriu a aplicação da metodologia DEA (Data Envelopment Analisys) em uma das etapas de um processo de benchmarking. Devido à natureza do método, que calcula as eficiências operacionais de um modelo formulado a partir das características do sistema estudado, o conteúdo do processo de benchmarking passou a ser essencialmente quantitativo até a fase de definição dos alvos de desempenho.

O conjunto integrado benchmarking-DEA foi proposto para detectar as melhores práticas operacionais das unidades de refino da empresa estudada, a partir de um modelo que considerava quatro parâmetros representativos deste tipo de segmento: o input "Capacidade Ociosa" e os outputs "Percentual de Derivados Leves", "Receita $/ \mathrm{m}^{3}$ de Óleo Processado" e "Produção de Gasolina A/100 $\mathrm{m}^{3}$ de Óleo Processado". Estes dados reais coletados na literatura foram executados pelo software Frontier Analiyst. Os resultados analisados foram considerados satisfatórios e permitiram que a implementação do estudo fosse bem-sucedida.

$\mathrm{O}$ estudo detectou as unidades mais eficientes do sistema, as refinarias R-6 e R-8. Estas unidades apresentaram as melhores práticas em relação ao modelo formulado, e serviram como referência para o estabelecimento de alvos de desempenho às demais unidades que não alcançaram 100\% eficiência. Estes alvos são quantitativos e deram origem à elaboração de um Plano de Melhorias. Este plano é composto de ações qualitativas (operacionais, de projeto e corporativas), capazes de tornar a unidade competitiva, promovendo-a de um estado de ineficiência para um estado de eficiência operacional. Há previsão de prazo e indicação de responsáveis pela execução do plano. As unidades onde as medidas podem ser executadas são identificadas em função da disponibilidade de seus recursos e dos seus esquemas de refino. Uma medida de melhoria somente é vinculada à uma unidade de refino, se tal medida for considerada factível de ser implementada. 
Em relação aos resultados encontrados pelo método DEA, é recomendável uma análise crítica calcada na experiência de profissionais especialistas do setor em questão. Neste sentido, foi realizada uma consulta a um da área com o intuito de verificar se as eficiências encontradas correspondem à realidade. Sua análise considerou os resultados pertinentes. Observou-se também, que todas as unidades que dispõem do processo "fundo de barril" Coqueamento Retardado em seus esquemas de refino, apresentaram bom desempenho (R-5, R-1 e R-7 com 86,78, 92,06 e 99,76\% de eficiência operacional respectivamente), mas havia uma expectativa de que alguma delas se localizasse na fronteira de eficiência máxima, o que não foi confirmado.

A ausência da DMU R-7 nesta fronteira também causou uma certa surpresa, pois trata-se da refinaria que detém as maiores produções de derivados e receitas anuais. Porém, apesar de seu desempenho operacional não ter alcançado o patamar de $100 \%$ de eficiência, esta DMU apresentou um excelente resultado ( $99,76 \%$ de eficiência), o que pode ser confirmado pelo fato desta ser a unidade que precisa investir menos para atingir a eficiência plena.

Com relação às DMU's R-6 e R-8, que apresentaram eficiência máxima, pode-se afirmar que a modelagem do problema, que minimizou as diferenças de escala ao considerar as produções de derivados leves e de gasolina A por volume de óleo processado, contribui positivamente para o alcance deste resultado. Este fato pode ser encarado como um fator positivo do modelo proposto, já que a eficiência operacional na utilização dos recursos disponíveis para o alcance de bons resultados, independe da magnitude ou da escala de produção da unidade de refino em questão. A DMU R-8 apresenta um grande equilíbrio em relação aos valores de cada parâmetro do modelo e, por este motivo, é uma unidade de referência para as consideradas ineficientes. Quanto à DMU R-6, seu resultado foi alavancado pelo expressivo valor do seu parâmetro "Receita $/ \mathrm{m}^{3}$ de Óleo Processado" $(222,19$ US\$), muito superior às demais DMU's. Vale lembrar que este é o output mais relevante para o modelo, de acordo com as restrições aos pesos.

O objetivo do estudo foi concluído com a elaboração do Plano de Melhorias. A execução deste Plano não foi realizada, pois não faz parte do escopo do trabalho, já que não houve aplicação prática em campo. É importante ressaltar que o referido plano traz medidas técnicas ou operacionais e, para que estas se tornem factíveis, ele deve ser acompanhado de um estudo mais específico de mercado.

Portanto, as principais contribuições deste trabalho foram um novo emprego da metodologia DEA como indicadora de desempenho operacional em um processo de benchmarking e a implementação do estudo no segmento de refino da empresa estudada, pois não há registro na empresa da utilização desses recursos para mensuração de eficiência operacional ou análise de competitividade.

\section{Referências Bibliográficas}

(1) Banker, R.D.; Charnes, A. \& Cooper, W.W. (1984). Some Models for Estimating Technical and Scale Inefficiences in Data Envelopment Analysis. Management Science, 30(9), 1078-1092.

(2) Calôba, G.M. (2003). Combinando Envoltória sob Ótica Dupla, Teoria da Preferência e Análise de Investimentos para Avaliação de Blocos Exploratórios de Petróleo e Gás no Leilão da ANP. Tese de M.Sc, COPPE/UFRJ, Rio de Janeiro, RJ, Brasil. 
(3) Charnes, A.; Cooper, W.W. \& Rhodes, E. (1978). Measuring the Efficiency of Decision-Making Units. European Journal of Operational Research, 2, 429-444.

(4) Homburg, C. (2001). Using Data Envelopment Analysis to Benchmark Activities. Int. J. Production Economics, 73, 51-58.

(5) Lins, M.E. \& Meza, L.A. (1999). Fronteiras de Produtividade. $1^{\mathrm{a}}$ ed., Universidade Federal do Rio de Janeiro, Rio de Janeiro.

(6) Paradi, J.C.; Rehm, S. \& Schaffnit, C. (1997). Performance Analysis for Engineering Teams at Bell anada. Centre for Management of Technology and Entrepreneurship, University of Toronto.

(7) Post, T. \& Spronk, J. (1999). Performance Benchmarking Using Interactive Data Envelopment Analysis. European Journal of Operational Research, 115, 472-487.

(8) Silva, H.B.F. (1994). Um Modelo Baseado na Análise de Encapsulamento de Dados (Data Envelopment Analysis - DEA) para Benchmarking. Tese de D.Sc, COPPE/UFRJ, Rio de Janeiro, RJ, Brasil.

(9) Ross, A. \& Droge, C. (2002). An Integrated Benchmarking Approach to Distribution Center Performance Using DEA Modeling. Journal of Operational Management, 20, 19-32.

(10) Spendolini, M.J. (1994). Benchmarking. $1^{\mathrm{a}}$ ed., Makron Books do Brasil, São Paulo.

(11) Vasconcellos, V.A. (2003). Detecção das Melhores Práticas Operacionais Através do Conjunto Benchmarking-DEA: o Caso das Refinarias de Petróleo. Tese de M.Sc, COPPE/UFRJ, Rio de Janeiro, RJ, Brasil. 